\title{
Supporting Mobile Activities in a Shared Semantic Ambient
}

\author{
Fabio Pittarello, Augusto Celentano \\ Università Ca' Foscari di Venezia, Dipartimento di Informatica \\ Via Torino 155, 30172 Mestre (VE), Italy \\ (pitt, auce) @unive.it
}

\begin{abstract}
We discuss an approach for modeling human activities in complex real environments, considering the delivery of services as a function of the semantic features of the environment and of the interaction between the users and their social networks. We propose an architecture supporting such a model, and discuss a case study about cooperative learning in a cultural heritage site.
\end{abstract}

\section{Introduction}

The execution of complex activities in wide and diversely structured environments is well supported by systems aware of the user and environment context, able to change their behavior to best fit the specific situation. Context aware adaptive systems are becoming common, and standards for describing the context are being consolidated [1-3]. Such systems are modeled according to two main paradigms: (1) systems that adapt the behavior of an application, designed to be adaptable, to a set of parameters that define the specific context $[4,5]$; and (2) systems that build an instance of an application by searching and integrating services appropriate for the specific context $[6,7]$. A common aspect of the two paradigms is the analysis of the "situation" and the choice of an appropriate set of actions. The choice is driven by parameters bound to the user, the devices, the network, the ambient, the location, the time, etc..

This paper discusses an approach for modeling human activities in complex real environments, considering the delivery of services as a function of the semantic features of the environment and of the interaction among the users and their social networks. The adaptation of services based on a set of independent parameters may be insufficient for activities executed in complex environments, composed of several tasks logically organized, extended in time and requiring cooperation between the users. In such cases, context adaptation must rely on the semantics of the whole application and of the ambients in which it is executed. As an example, let's examine the delivery of several types of information about an urban area. Traditionally, adaptation could specialize the search and presentation of information according to the user device, giving:

- on a conventional Internet access, full search capabilities with a multimedia presentation of usually limited quality to accommodate also for poor network performances; 
- on a location specific kiosk, restricted search capabilities constrained to the ones relevant in the location, with full multimedia presentation since the multimedia material can be locally stored;

- on a PDA, information abstraction and progressive disclosure on demand, taking into account both the limitations of the device and the bandwidth and costs of the wireless network;

- on a cellular phone, limited, guided search capabilities with short vocal or text messages.

Shifting from context awareness to ambient awareness, services are delivered not only as a function of local properties of the user and her/his environment, but also according to the ambient general properties and role, i.e., according to the ambient semantics. The example above could therefore be discussed with a different perspective about the variants needed for an on-line information service. Assuming that conventional Internet access to a Web server through a personal browser can be tailored to the user context as discussed above, other ambients may offer the same service in different ways:

- in a public place, such as a mall, a station, a square, only general info of public interest is delivered through large displays and unattended kiosks;

- ambients characterized by a specialization of their functions deliver only information related to the function. For example, in a theatre atrium information about the theatre program is displayed, while in a museum information pertinent to the museum is given;

- in some ambients (e.g., a church) the specificity of the function and the mood of the place can suggest to inhibit at all information delivery;

- finally, the modality of delivery, e.g., audio messages or video displays, can also be bound to the average user attention in the ambient, for example by delivering audio messages in a station and video messages in a mall.

An additional level of adaptation comes from the knowledge of the user social environment, which may extend the information available to the user and his/her capability to perform a task, setting up a cooperation among the user and his/her network of contacts. Considering the user social network improves the set of available services, providing additional information by integrating the communication in the user social network with the results of the local services.

\section{Modeling activities in semantic spaces}

We make some assumptions, without entering into detailed specifications:

- the physical ambient in which user activities take place is part of an urban environment, whose topological and geometric features are known, and can be classified according to a suitable ontology [8];

- the users are primarily interested to navigating the ambient for discovering (or receiving on explicit query) information, possibly in multimedia format, through multimodal interaction; 
- the ambients in which users navigate have distinguished roles in terms of purpose and social relations. For example, they can be public or private ambients (e.g., a square vs. an apartment) according to some access control policy, or can be connection or action spaces (e.g., in a museum, an aisle vs. an exhibition room), according to the absence or presence of specific activities other than movement.

- the user activities are not isolated, but are elements of a plan (which can be completely defined or dynamically built) mixing activities and navigation in the environment, and requiring the execution of a number of different tasks [9];

- finally, the users themselves are not isolated, but immersed in a network of social relations which allow (and may in some cases require) interaction among the participants in order to execute the activities.

The semantic description of the environment, possibly mapped to its physical description (e.g., through a corresponding 3D geometry) is necessary for presenting the users a multimodal description of the areas that compose the physical environment, and of the appliances the user can interact with. Cues for moving in the environment and interacting with it, given according to a hierarchy of environment locations, are more effective if the user understands the relations between the environment topology and the meaning of its components, such as rooms, places, stairs, etc. [10,11].

The semantic description can also answers specific information needs: e.g., in a cultural tourism application a user, during a guided tour, might be interested in the architectural details of a building, or in the logical organization of the work of arts contained in a suite of exhibition rooms.

Cooperation among users can be exploited in two ways: locally and remotely. Local cooperation takes places in the environment and its surroundings. Several users may be involved in the same activity, or can exchange mutual help to improve a task execution. The network of social relations of a user in practice extends its environment with parts of the environments of the cooperating users.

\section{Applications, activities and services}

Generally speaking, we can define an application as a set of cooperating services executing tasks and exchanging data, providing computational and information support to human activities in a coordinated plan of actions. We do not assume any specific service architecture, such as Web services, but remain at an abstract level. Services execute (sub-)tasks, receive and deliver information and objects, process information content and presentation, etc. according to some plan. Services may also be executed by humans: for example, an information delivery service can be offered, in different contexts, by an automatic answering system, an interactive kiosk or a human guide.

Different types of services are offered to users populating an ambient: field services and local services are characterized by being, respectively, accessible in 
a wide area, possibly by more users at the same time, or at a specific location, usually by one user at a time. Individual and social services are characterized by being, respectively, executed by a single user or cooperatively by a set of users.

Field services. Field services are associated to a part of the ambient geometrically defined as an area, e.g., a street, a square, a room. They are accessible, in principle, from every location inside the area. They are not bound to physical contact with the user, and do not perform transmission of concrete objects, such as tickets, money and goods. Examples of field services are:

- information delivery services in the waiting room of a railway station through large displays, visible from every room point;

- audio messaging services through loudspeakers unconditionally directed to the public in the ambient;

- services delivered through wireless communication technologies such as WiFi of mobile telephony, possibly contextualised on the user location.

In general, the service dealer location and the user location inside the field is not relevant for the service execution, even if different locations can affect the quality and the performance of the service.

Local services. Local services are associated to an appliance of the environment, a physical or virtual artifact which contains the interface between the user and the service. They are accessible only in proximity of the appliance, which acts as the service dealer, may be bound to a physical contact between the user and the appliance, and may exchange concrete objects, such as tickets, money, goods. Examples of local services are:

- ticketing services delivering concrete tickets (as opposed to ticket reservation services), possibly as the final step of a sequence of reservation-issuingdelivering services;

- ATM services, and in general services related to physical exchange of money or goods;

- access control services, which are executed at specific site entrances;

- services based on proximity identification, such as Bluetooth or RFID based services, active close to an object or a location.

Individual services. Individual services are activated by a user as part of an application requiring neither cooperation nor sharing with other users. Local services are often individual services, and the effect of their execution is usually limited to the user that has executed the service. A typical example is an ATM service.

Social services. Social services are executed by a user in cooperation with other users, which can be located locally or remotely. Cooperative services are often field services, due to the need of sharing the service access from the different places where the users are. They could also be executed as the result of a local service, whose completion requires (as a subtask) the access to a wider community, through remote communication tools. An example of social service is the 
search for information needed to complete the execution of a task, which may require the intervention of other users expert of the task.

\section{A case study: cooperative learning in a cultural heritage site}

The following example illustrates how the integrated knowledge of an ambient, services and social network can be used in the context of a cooperative learning experience. The case study considered is that of a class of students visiting a cultural heritage site for an on-the-field learning experience. Students are divided into small groups and the task of each group is to compose an assignment related to a specific topic, e.g., expressionist paintings. Each group is provided with a mobile device as a support for completing the task.

The knowledge of the physical features of the ambient and of the information associated to its components constitutes a first layer that can be used by an implementation architecture for helping the students to find and reach specific locations compliant with the goal of the initial task [11]. Once in the correct location, the student can access information related to the surrounding artworks. Group discussion is catalyzed by this information level and leads to the production of a preliminary version of the assignment that is the result of the local discussion (i.e., in the group).

Visiting a cultural heritage site implies the access to a set of resources that can be available with limited spatial and temporal constraints, but also to resources that can be conditioned by such limitations: for example, a pavilion with a specialized installation could be accessed only by one person at a time, or a video could be displayed in a room with a very small number of seats. In such situations the knowledge of a network of services for mapping such resources, monitoring and reserving them can be useful for optimizing the time available to students for completing their task.

When the information associated to the environment has already been examined without giving the users a satisfactory level of knowledge, the access to external networks, such as communities and even the web, can be useful to identify additional local (e.g., another class of students or an expert currently visiting the same exhibition) or remote (e.g., people whose profile is compliant with the students needs and that are available for lending a hand) individuals that could give additional help.

The knowledge of the environment, and in particular of the environment the group of students are currently visiting, is used as an additional cue for finding adequate support in the social network, informing the available remote human resources about the context of the group, giving it a better support.

Such support is in fact an enhancement of the activities characterizing the online discussion groups, where the participants usually have to explain not only their problems, but also the context where they are; the context is a useful information for the remote users trying to help them. For example, a remote user knowing about the museum in which the group is working, could make a 


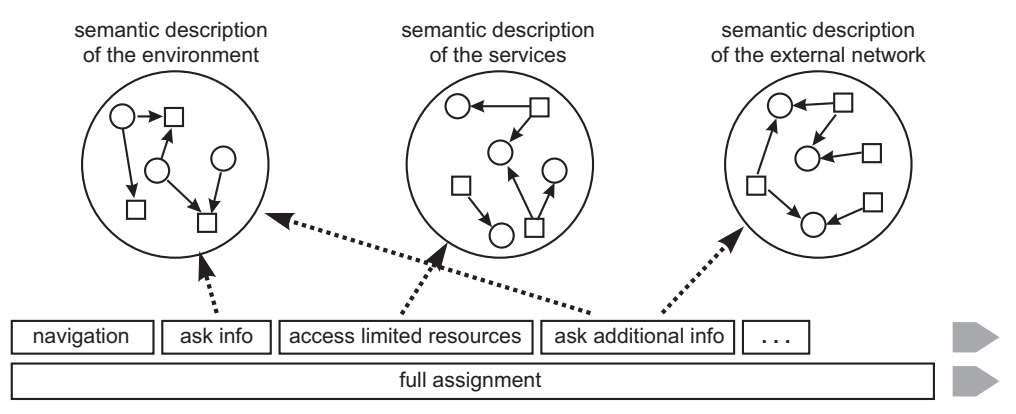

Fig. 1. Activity, ambient, service and network relations

precise suggestion, such as: "Look at room 5, next to your room, where artworks produced by the masters of the Bauhaus art school are displayed. It could be useful to compare them with the expressionist works you're considering", while a user unaware of the specific group context could only give a generic support independent of the museum content.

\section{Relating activities to ambients and to social networks}

Figure 1 shows a scheme of the task the group has been assigned to and the relations with the different semantic descriptions that are accessed in order to reach the goal. The task has been split in several subtasks, shown in the figure, that are required to complete the assignment. For the sake of simplicity Figure 1 describes a specific sequence of tasks and their relations with the semantic descriptions. While the relations between a given subtask type and a specific semantic description are fixed, the different subtasks can be iterated and can be organized in different sequences, according to the interaction patterns determined by the users' actions. Figure 1 evidences several relations:

- users moving around to find right places and artworks (navigation task) take advantage of a semantic description of the environment while moving; written or spoken hints are presented on the mobile device for guiding the users to the relevant locations, such as "go straight for 3 meters, turn right and reach the hall with a circle of columns in the center";

- users searching for information about museum spaces and artworks (ask info task) require also the access to the semantic description of the environment and to the associated multimedia information, such as information associated to the semantic object Alter Klang by Paul Klee, belonging to the class artwork, or to the semantic space Early Expressionism Room belonging to the class room, for receiving a first set of information that they can use for their assignment;

- users activating the access limited resources task take advantage of the semantic description of services; such knowledge is used by the users for accessing the services and exchanging information about their availability and 


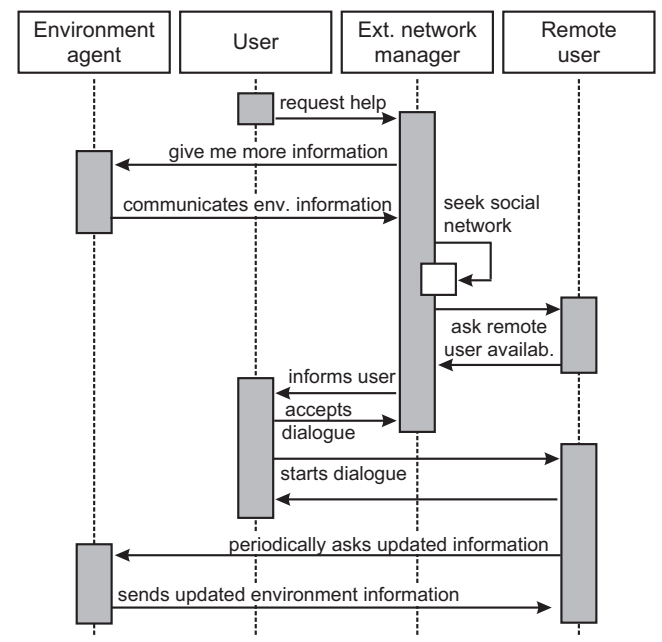

Fig. 2. Communication between a user and the external network

reservation (e.g., "reserve 4 seats for the projection about Franz Marc starting at 10.00 a.m.");

- users executing the ask additional info task can take advantage of an external network of resources in order to complete their task, using both multimedia resources available on the web (such as search engines, on-line encyclopedias, dictionaries, etc.) and human resources that are selected on the semantic representation of the social network on the basis of the users' profiles and other parameters.

The access to the external network represents the most complex situation and includes also the access to the semantic representation of the environment for assisting the user. Figure 2 shows a sample of the communication protocol between the different subjects in the external network. While the network is usually accessed to get multimedia resources available on the web, the example is focused on the social network that represents the human component of the external help resources.

The access to the social network is triggered by a request for additional info, initiated by the group of students through their mobile device. Such request is received by a software agent, the external network manager, that seeks the semantic description of the social network using as input the students' profiles, the keywords related to the topics requested by the students and the description of the environment the students are currently visiting. The environment information is asked to another agent, the environment agent, that sends a machinereadable description of the part of the environment the subjects are currently visiting. Such information is processed by the environment agent on the basis of the semantic description of the environment and on the specification of the current location of the users, identified through their mobile device. Such in- 
formation represents an enhancement of the context associated to the students because it includes an explicit description of the environment context that may have been omitted or only partially described in the students' requests.

The external network manager matches all such information with the semantic description of the social network, in order to find the potential helpers. Generally speaking, such human resources have declared in their profile the permission to be requested for help about certain topics by specific categories of users. They are divided in local and remote users on the basis of their location in relation to the location of the people requiring help. A different communication protocol is activated according to the helpers' location. The example of Figure 2 considers the case of a remote user (a helper far from the students, who therefore can only interact with them by sending information to their mobile device) who is contacted by the external network agent to confirm his/her current availability. The network manager, on the basis of a positive acknowledgement, notifies the remote user profile to the students that may decide to start the dialogue. The communication happens primarily between the group of students and the remote user that may freely decide the contents and the duration.

A complementary support role is offered by the environment agent that can be periodically queried (upon the students' group permission) by the remote user for sending updated information about the users' environment. Such information can be profitably used by the remote users for giving a better support, since the knowledge of the part the museum the students they are currently visiting can guide the group to interesting local resources.

\section{An architecture for mobile activities in shared ambients}

Figure 3 illustrates the functional architecture of a system supporting mobile activities in shared ambients. Services are distributed in the environment as local or field services. The same service can be executed in different ways under different contexts. Also, different services can be available to users under different contexts. Therefore, the visibility of services and their access is mediated by a semantic description (service ontology), which manifests to the user only a set of qualified services, i.e., services which can be properly executed in the user and ambient context.

The ambient is described at two levels, both as a base geometry and at a semantic level, obtained by filtering the base geometry through an ambient ontology which gives meanings and roles to the objects and places of the ambient, defining also the relationships between places and objects [11]. Each place and each ambient object can also be linked to a set of multimedia/multimodal information (not shown in Figure 3 for simplicity), which is delivered by proper services through the suitable and context compliant communication channel when information services are required.

The semantic representation of the ambient is matched against the collection of qualified services, providing the set of services and the related communication channels that are compatible with the semantics of the ambient. The user receives 


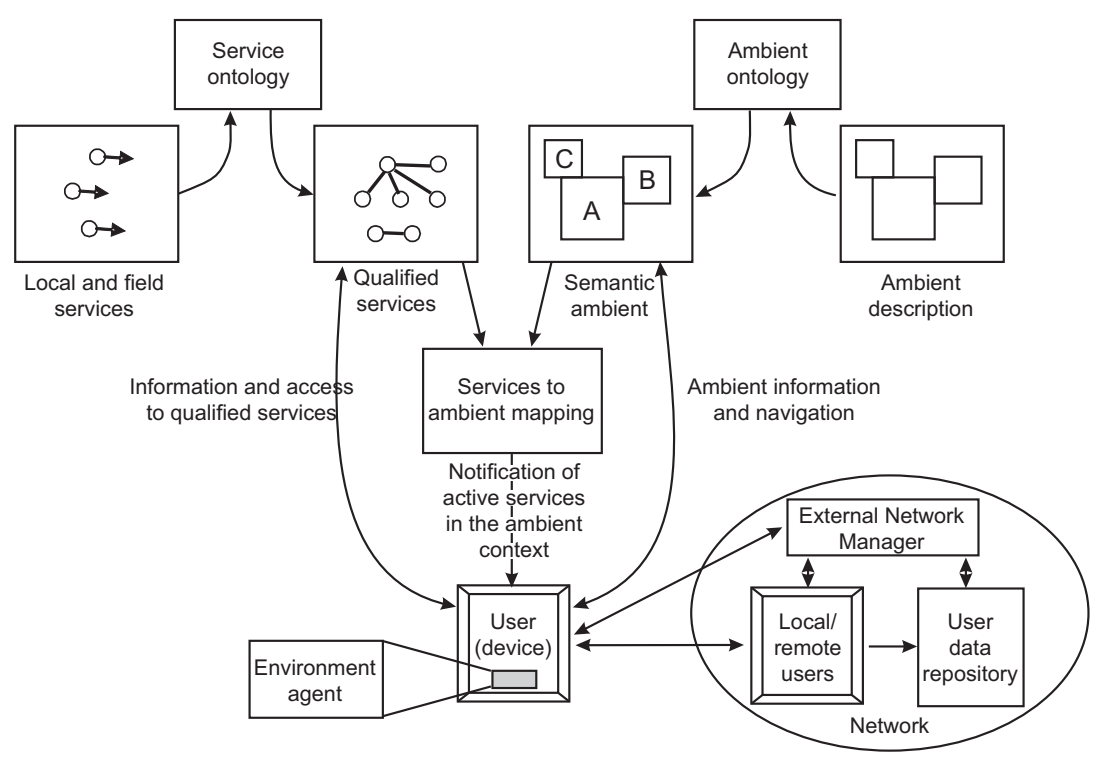

Fig. 3. An architecture for mobile services in shared ambients

on his/her device information about the available services, depending on the location, the semantic of the place, his/her own profile and history (collected by sensors and interaction patterns) and the device itself. The user can then access the available services.

The user can also access a network of external relations, where remote users may be queried for support. Access is managed by the external network manager that seeks the potential helpers in the user data repository, filled with data coming from people that has accepted to publish them. The manager asks to the potential helpers and to the user a confirmation of their availability for starting a dialogue; on a positive acknowledgment, a direct communication channel between the users is opened. Alternatively, if the helpers are close to the user location, the manager may notify them of such situation for a direct contact. In the case of remote communication, the channel opened by the external network manager can be used by the helpers also for accessing the environment agent, a software component embedded in the user device that knows information related to the environment, the qualified services and the user data (e.g. location). As explained in Section 5, such information can be useful to remote helpers for improving their assistance to the user.

\section{Conclusions}

In this paper we have proposed an approach to the design of mobile pervasive services based on three key elements: (1) the semantic characterization of the ambients where services can be accessed; (2) the semantic description of services 
matching the services available in the environment, active at a given moment, with the constrains for their delivery according to different context parameters; (3) the opening to an external network of information and remote users to improve the number and quality of available services.

The semantic knowledge of the different ambient components is cooperatively used to reach several goals: the knowledge of the environment and of the services is used to give a first level of support to the user activities; the knowledge of the user social network is used to find additional support; finally the knowledge of the user ambient is shared to give to the components of the social network additional cues for supporting the demanding user.

Our next step will be the design of a more integrated cooperation between the available services and the social network, with the aim of adapting the services according to the information provided by the user contacts. For example, user preferences might be inferred from community profiles, and hints provided by other users in blogs and forums might be used to select and adapt the more suitable services.

\section{References}

1. Chen, G., Kotz, D.: A survey of context-aware mobile computing. Technical Report TR2000-381, Dartmouth College, Department of Computer Science (2000)

2. Dey, A.K.: Understanding and Using Context. Personal Ubiquitous Computing 5 (2001) 4-7

3. Held, A., Bucholz, S., Schill, A.: Modeling of context information for pervasive computing applications. In: Proc. SCI2002, 6th World Multiconference on Systemics, Cybernetics and Informatics, Orlando,FL (2002)

4. Lemlouma, T., Layaïda, N.: Context-aware adaptation for mobile devices. In: Proc. IEEE Int. Conf. on Mobile Data Management. (2004) 106-111

5. Villard, L., Roisin, C., Layaïda, N.: An XML-based multimedia document processing model for content adaptation. In King, P., Munson, E.V., eds.: Digital Documents: Systems and Principles. Volume 2023 of LNCS. Springer (2000) 104119

6. Sycara, K., Paolucci, M., Ankolekar, A., Srinivasan, N.: Automated discovery, interaction and composition of semantic web services. Journ. Web Sem. 1 (2003) $27-46$

7. Vukovic, M., Robinson, P.: Adaptive, planning-based, web service composition for context awareness. In: Proc. Int. Conf. on Pervasive Computing, Vienna, Austria (2004)

8. Bille, W., Troyer, O.D., Kleinermann, F., Pellens, B., Romero, R.: Using ontologies to build virtual worlds for the web. In: Proc. ICWI, IADIS International Conference WWW/Internet, Madrid, Spain (2004) 683-690

9. Pittarello, F., Fogli, D.: Modelling complex user experiences in distributed interaction environments. In: Proc. DMS, 11th Int. Conf. on Distributed Multimedia Systems, Banff,Canada (2005)

10. Mansouri, H.: Using Semantic Descriptions for Building and Querying Virtual Environments. PhD thesis, Vrije Universiteit Brussel (2005)

11. Pittarello, F., Faveri, A.D.: A semantic description of 3 D environments: a proposal based on Web standards. In: Proc. Web3D 2006, Columbia, Maryland (2006) 85-95 